\title{
Collapse of the turbulent dissipative range on Kolmogorov scales
}

\author{
R. A. Antonia, ${ }^{1}$ L. Djenidi, ${ }^{1}$ and L. Danaila ${ }^{2, a)}$ \\ ${ }^{1}$ School of Engineering, University of Newcastle, NSW 2308, Australia \\ ${ }^{2}$ CORIA, Avenue de l'Université, BP 12, 76801 Saint Etienne du Rouvray, France
}

(Received 22 August 2013; accepted 9 March 2014; published online 16 April 2014)

\begin{abstract}
It is pointed out that the collapse of the turbulent dissipative range on Kolmogorov scales does not require either of the two major assumptions in Kolmogorov's ["The local structure of turbulence in incompressible viscous fluid for very large Reynolds numbers," Dokl. Akad. Nauk USSR 30, 299 (1941)] similarity hypothesis, i.e., $R_{\lambda}$, the Taylor microscale Reynolds number, is very large and local isotropy is satisfied. In particular, the Kolmogorov velocity and length scales are shown to be the appropriate normalization scales when the large-scale terms in the transport equations for the second-order statistics can be neglected. Evidence for this scaling is discussed critically on the basis of the available data. It is also shown that this scaling breaks down when $R_{\lambda}$ becomes too small, typically below 20. 2014 AIP Publishing LLC. [http://dx.doi.org/10.1063/1.4869305]
\end{abstract}

\section{INTRODUCTION}

Much attention has been given to the study of the turbulent small-scale motion (or SSM), mainly as a result of the postulates made by Kolmogorov. The first two similarity hypotheses widely known as K41 were enunciated by Ref. 1. The present paper is concerned with K41, which essentially postulates that the statistics of the small-scale motion are determined uniquely by two parameters: $\langle\epsilon\rangle$, the mean energy dissipation rate and $v$, the kinematic viscosity. Two assumptions underpin K41: the Reynolds number is "very large" and the small scales are isotropic. Neither of these is likely to hold for the overwhelming majority of flows encountered in the laboratory.

It has now been established unambiguously that the finite Reynolds number (FRN) effects cannot be discounted, especially on inertial range (or IR) scales (e.g., Refs. 2-8). Starting with Lin's transport equation for the $3 \mathrm{D}$ energy spectrum $E(k, t)$, viz.,

$$
\frac{\partial E(k, t)}{\partial t}=T(k, t)-2 v k^{2} E(k, t)
$$

where $T(k, t)$ is the nonlinear spectral transfer function, Ref. 2 concluded that the Kolmogorov $4 / 5$ law, ${ }^{9}$ which is the only exact relation - unaffected by intermittency effects - for inertial range statistics, is unlikely to be observed in the laboratory, when $R_{\lambda}$ is smaller than about $10^{3} . R_{\lambda}$ is the Taylor microscale Reynolds number $\left(R_{\lambda}=\left\langle u^{2}\right\rangle^{1 / 2} \lambda / \nu, \lambda\right.$ is the Taylor microscale associated with the longitudinal velocity fluctuation $u$ ). Qian also concluded that $R_{\lambda}$ should be greater than $10^{4}$ for the extent of the inertial range to be about one decade and emphasized that the so-called inertial range found in experiments and simulations is only a scaling range which, strictly, should be distinguished from Kolmogorov's inertial range. These conclusions have since been convincingly backed up by results from methods in either physical space, see Eq. (3) in Sec. II, or in Fourier space, using Eq. (1), albeit with some differences in the precise value of $R_{\lambda}$ needed to establish a substantial inertial subrange. Using the former approach, Ref. 7 found that the asymptotic value of $4 / 5$ is approached more rapidly for forced than for decaying turbulence and estimated that an inertial range will exist

\footnotetext{
a)Electronic mail: danaila@coria.fr
} 
only if $R_{\lambda}$ exceeds about $10^{3}$ with forcing and about $10^{6}$ in decaying turbulence. More recently, Ref. 8 who used Lin's equation and the Eddy Damped Quasi Normal Markovianisation closure found that $R_{\lambda}$ should exceed about $5 \times 10^{3}$ for forced turbulence and $5 \times 10^{4}$ for freely decaying turbulence.

The SSM contains scales in both the dissipative range (DR) and the IR or, more appropriately, scaling range (SR). As outlined above, the SR scales are affected by the FRN effect. This effect, which becomes more pronounced as the scale increases, reflects the importance of the non-stationary term in the Kármán Howarth ${ }^{10}$ (or KH) equation (see Sec. II) or, equivalently, Eq. (1).

The present paper focuses on the DR scales where the effect of the large scales tends to be small. As detailed in Secs. II and III, the neglect of this effect leads to a possible similarity solution based on Kolmogorov scales, irrespectively of whether the flow is locally isotropic (Sec. II) or locally axisymmetric (III). This in turn suggests that the two main requirements of K41, viz., $R_{\lambda}$ should be very large and the small scales should be isotropic, could be relaxed, provided the large-scale effects are negligible. Available one-dimensional spectra are discussed in some detail in Sec. IV in the context of this relaxation. In Sec. V, we consider specific situations where the Kolmogorov scaling in the dissipative range is no longer tenable. Section VI summarizes our conclusions.

\section{SIMILARITY SOLUTION FOR ISOTROPIC SMALL SCALES}

The analytical approach is developed by considering the transport equations in physical space of the second-order statistics (structure functions). Obviously, a similar approach can be developed in Fourier space. The crux of the analytical approach is a critical comparison between terms that should be prevalent in the DR and terms which reflect various types of effects associated with large scales, such as the decay of turbulent energy, e.g., decaying homogeneous isotropic turbulence for which local isotropy is tenable even though $R_{\lambda}$ may be small. This case, which has been widely studied in the literature, is reanalyzed in this section.

An appropriate starting point is the transport equation for the two-point velocity correlation function first derived by KH for homogeneous isotropic (at all scales) turbulence, i.e.,

$$
\begin{aligned}
\frac{\partial}{\partial t}\left(\left\langle u^{2}\right\rangle h\right) & =\frac{\partial}{\partial r}\left(\left\langle u^{2}\right\rangle^{3 / 2} k\right)+4 \frac{\left\langle u^{2}\right\rangle^{3 / 2} k}{r} \\
+ & 2 v\left[\frac{\partial^{2}\left\langle u^{2}\right\rangle h}{\partial r^{2}}+\frac{4}{r} \frac{\partial\left\langle u^{2}\right\rangle h}{\partial r}\right],
\end{aligned}
$$

where $h\left(=\langle u(x) u(x+r)\rangle /\left\langle u^{2}\right\rangle\right)$ and $k\left(=\left\langle u^{2}(x) u(x+r)\right\rangle /\left\langle u^{2}\right\rangle^{3 / 2}\right)$ are the 2-point second- and third-order velocity correlation functions; the notation used here differs from that in $\mathrm{KH}$ and Ref. 11 .

The corresponding transport equation for the second-order velocity structure function was written first by Ref. 9 and later discussed in more detail by Ref. 11. Reproduced below is the form of the equation considered by Batchelor, viz.,

$$
\begin{array}{r}
3 \frac{\partial}{\partial t}\left(2\left\langle u^{2}\right\rangle-\left\langle(\delta u)^{2}\right\rangle\right)=\frac{\partial}{\partial r}\left\langle(\delta u)^{3}\right\rangle+4 \frac{\left\langle(\delta u)^{3}\right\rangle}{r} \\
-6 v\left[\frac{\partial^{2}\left\langle(\delta u)^{2}\right\rangle}{\partial r^{2}}+\frac{4}{r} \frac{\partial\left\langle(\delta u)^{2}\right\rangle}{\partial r}\right]
\end{array}
$$

where $\left\langle(\delta u)^{2}\right\rangle=\left\langle(u(x+r)-u(x))^{2}\right\rangle$ and $\left\langle(\delta u)^{3}\right\rangle=\left\langle(u(x+r)-u(x))^{3}\right\rangle$ are the second- and thirdorder structure functions, respectively. The relations between the structure functions and correlation functions are

$$
\left\langle(\delta u)^{2}\right\rangle=2\left\langle u^{2}\right\rangle(1-h(r))
$$

and

$$
\left\langle(\delta u)^{3}\right\rangle=6\left\langle u^{2}\right\rangle^{3 / 2} k(r)
$$


The term on the left side of Eq. (3) reduces to $-4\langle\epsilon\rangle$ if $\partial\left\langle(\delta u)^{2}\right\rangle / \partial t$ is zero, since

$$
\langle\epsilon\rangle=-\frac{3}{2} \frac{d\left\langle u^{2}\right\rangle}{d t} .
$$

After integrating Eq. (3) with respect to $r$ and applying the boundary conditions

$$
\left\langle(\delta u)^{3}\right\rangle=0,
$$

at $r=0$, Eq. (3) becomes (Kolmogorov), ${ }^{9}$

$$
-\frac{4}{5}\langle\epsilon\rangle r=\left\langle(\delta u)^{3}\right\rangle-6 v \frac{d\left\langle(\delta u)^{2}\right\rangle}{d r} .
$$

Batchelor showed that a similarity solution of Eq. (5) is given by

$$
\begin{aligned}
& \left\langle(\delta u)^{2}\right\rangle=(\nu\langle\epsilon\rangle)^{1 / 2} f\left(\frac{r}{\eta}\right), \\
& \left\langle(\delta u)^{3}\right\rangle=(\nu\langle\epsilon\rangle)^{3 / 4} g\left(\frac{r}{\eta}\right),
\end{aligned}
$$

i.e., the similarity scales are the Kolmogorov velocity $u_{K}=(\nu\langle\epsilon\rangle)^{1 / 4}$ and length $\eta=\left(\nu^{3} /\langle\epsilon\rangle\right)^{1 / 4}$ scales. Equation (5) then becomes

$$
-\frac{4}{5} \frac{r}{\eta}=g\left(\frac{r}{\eta}\right)-6 v \frac{d f}{d(r / \eta)},
$$

where each term is a function only of $r / \eta$. This "prompted" Batchelor to suggest that Eq. (7) may be used to support Kolmogorov's first similarity hypothesis. ${ }^{9}$ Kolmogorov and Batchelor neglected the $\partial / \partial t$ term by assuming stationarity, an assumption that becomes more readily justifiable as $R_{\lambda}$ keeps increasing. Batchelor argued that when the focus is on small eddies within a domain of spatial extent $G$, it is not unreasonable to assume that the motion due to these eddies is approximately steady for time intervals within $G$. Given that the characteristic period of an eddy should decrease as the size of the eddy decreases, it should become increasingly smaller compared to the characteristic time $L /\left\langle u^{2}\right\rangle^{1 / 2}$ of the flow, where $L$ is the integral length scale. A similar argument was proposed by Ref. 12.

Saffman ${ }^{13}$ pointed out that, for homogeneous turbulence, $R_{\lambda}$ does not necessarily have to be large for the inequality

$$
\frac{\partial\left\langle(\delta u)^{2}\right\rangle}{\partial t} \ll \frac{4}{3}\langle\epsilon\rangle
$$

to be satisfied. If $t$ is associated with a characteristic time scale $\left(L /\left\langle u^{2}\right\rangle^{1 / 2}\right)$ of the large scale motion, then

$$
\frac{\left\langle(\delta u)^{2}\right\rangle}{u_{K}^{2}} \ll \frac{4}{3}\left(\frac{L}{\left\langle u^{2}\right\rangle^{1 / 2}}\right) \frac{\langle\epsilon\rangle}{u_{K}^{2}} .
$$

Now, if $L=\lambda R_{\lambda} / 15$, the normalized dissipation rate parameter $C_{\epsilon}$, defined as

$$
C_{\epsilon}=\frac{\langle\epsilon\rangle L}{\left\langle u^{2}\right\rangle^{3 / 2}}
$$

is 1 , so that $\left\langle u^{2}\right\rangle /\langle\epsilon\rangle\left(=L /\left\langle u^{2}\right\rangle^{1 / 2}\right)$ can also be interpreted as a characteristic time scale of the large scale motion. With $\langle\epsilon\rangle=15 v\left\langle u^{2}\right\rangle / \lambda^{2}$, it follows that

$$
\frac{\left\langle(\delta u)^{2}\right\rangle}{u_{K}^{2}} \ll \frac{4}{3} \frac{R_{\lambda}}{15^{1 / 2}}
$$

or, alternately,

$$
\frac{\left\langle(\delta u)^{2}\right\rangle}{u_{K}^{2}} \ll \frac{4}{3} \frac{\left\langle u^{2}\right\rangle}{u_{K}^{2}},
$$


since $\left\langle u^{2}\right\rangle / u_{K}^{2}=2 R_{\lambda} / 15^{1 / 2}$. Relation (8) can therefore be satisfied either when $R_{\lambda}$ is sufficiently large or when the scale $r$ is sufficiently small $\left(\left\langle(\delta u)^{2}\right\rangle /\left\langle u^{2}\right\rangle \ll 1\right)$. In either case, the similarity solution

$$
\begin{aligned}
& \left\langle(\delta u)^{2}\right\rangle=u_{0}^{2} f\left(\frac{r}{l_{0}}\right), \\
& \left\langle(\delta u)^{3}\right\rangle=u_{0}^{3} g\left(\frac{r}{l_{0}}\right),
\end{aligned}
$$

with $u_{0}$ and $l_{0}$ as the relevant velocity and length scales, satisfies Eq. (5), viz.,

$$
\frac{u_{0}^{3}}{\langle\epsilon\rangle l_{0}} g\left(\frac{r}{l_{0}}\right)=\frac{4}{5} \frac{r}{l_{0}}-6 \frac{v u_{0}^{2}}{\langle\epsilon\rangle l_{0}^{2}} f^{\prime}
$$

(the prime signifies derivative with respect to $r / l_{0}$ ), provided that their non-dimensional coefficients are constant, namely,

$$
u_{0}^{3} /\langle\epsilon\rangle l_{0}=C_{1}
$$

and

$$
v u_{0}^{2} /\left(\langle\epsilon\rangle l_{0}^{2}\right)=C_{2} .
$$

A possible solution which satisfies (16) and (17) is $u_{0} \sim(\nu\langle\epsilon\rangle)^{1 / 4} \equiv u_{K}$ and $l_{0} \sim\left(v^{3} /\langle\epsilon\rangle\right)^{1 / 4} \equiv \eta$, i.e., the Kolmogorov velocity and length scales are the relevant similarity scales when inequality (8) applies. Another possibility is to first assume that the relevant similarity velocity scale is $u_{K}$, it then follows that the similarity length scale is $\eta$. Under these conditions, $C_{1}=1$. Note that if the similarity velocity scale $u_{0}$ is not $u_{K}$, then the similarity length scale cannot be $\eta$, and under these conditions, (16) becomes $R e_{l_{0}}=\frac{l_{0} u_{0}}{v}=$ const., i.e., the Reynolds number based on the similarity scales should be constant for the similarity at scale $l_{0}$ to be satisfied. Equation (15) does not require $R_{\lambda}$ to be large for the dissipative scales to satisfy Kolmogorov similarity.

We now examine the situation where the KH equation does not apply, since the effect of the large scale terms, due, for example, to the presence of the mean shear or turbulent diffusion, needs to be taken into account. As a first step, we assume that local isotropy still holds. Therefore, the transport equation for $\left\langle\left(\delta u_{i}\right)^{2}\right\rangle$ (summation applies to double Latin indices) becomes ${ }^{14}$

$$
\begin{array}{r}
-\frac{4}{3}\langle\epsilon\rangle r+2 v \frac{d}{d r}\left\langle\left(\delta u_{i}\right)^{2}\right\rangle-\left\langle\delta u\left(\delta u_{i}\right)^{2}\right\rangle \\
-\frac{1}{r^{2}} \int_{0}^{r} s^{2}\left[2 \frac{\partial U}{\partial y}\langle\delta u \delta v\rangle+\partial_{y}\left\langle\left(v+v^{+}\right)\left(\delta u_{i}\right)^{2}\right\rangle\right] d s=0,
\end{array}
$$

where $s$ is a dummy variable. An important remark needs to be made at this stage. Both additional terms in Eq. (18) reflect large-scale effects in a fixed frame; the first one is due to the weak shear, while the second one reflects the inhomogeneity along the direction $y$ normal to the wall (when one is present in the flow). Equation (18) is analogous to the scale-by-scale energy budget equation for temperature fluctuations fed by a mean temperature gradient. ${ }^{15}$ Furthermore, Eq. (18) may be written in the generic form

$$
\langle\epsilon\rangle F_{1}(r)+v F_{2}(r)+F_{3}(r)+L S T(r)=0,
$$

where $F_{i}$ are functions of the modulus $r$ of the increment vector $\vec{r}$ and eventually involve constants (e.g., $F_{1}(r)=-\frac{4}{3} r$, so that the first term in Eq. (19) becomes $\left.-\frac{4}{3}\langle\epsilon\rangle r\right)$. Term $F_{3}$ corresponds to the third-order term. It is important to note that the prefactors of the functions $F_{1}$ and $F_{2}$ are the physical parameters $\langle\epsilon\rangle$ and $\nu$. These are the only physical parameters which appear in the 1-point energy budget equation, i.e., the limiting form of (19) for scales larger than the integral scale. The term LST (or "Large-Scale Term") depends on $r$ and includes physical constants characteristic of the flow, such as the mean shear $\partial U / \partial y$ and turbulent diffusion.

Finally, the only necessary condition for Kolmogorov similarity is that LST becomes negligible at small scales with respect to the first two terms in Eq. (19) (term $F_{3}$ reflects turbulent advection 
and naturally vanishes for small scales). Under these conditions, Eq. (19) reduces to

$$
\langle\epsilon\rangle F_{1}(r)+v F_{2}(r) \approx 0,
$$

and the only similarity at these scales is based on length/velocity scales constructed from the physical parameters $\langle\epsilon\rangle$ and $\nu$. Kolmogorov scaling is thus the only solution tenable over the range of scales where large-scale effects may be neglected.

\section{SIMILARITY SOLUTION FOR LOCAL AXISYMMETRY}

The simplest relaxation of local isotropy is to assume local axisymmetry, or invariance of smallscale statistics with respect to rotation about a chosen coordinate axis or axis of axisymmetry. All statistics now depend on two variables: $\mathrm{r}$ (the modulus of the separation vector $\vec{r}$ ) and $\mu$, the cosine of the angle between the axis of axisymmetry and $\vec{r}$. The transport equation for the second-order structure function $\left\langle\left(\delta u_{i}\right)^{2}\right\rangle$ is ${ }^{16}$

$$
\begin{array}{r}
\partial_{t}\left\langle\left(\delta u_{i}\right)^{2}\right\rangle(\vec{r})+2\left\langle\delta\left(U_{\alpha} \partial_{\alpha} u_{i}\right) \cdot \delta u_{i}\right\rangle(\vec{r})+2\left\langle\delta\left(u_{\alpha} \partial_{\alpha} U_{i}\right) \cdot \delta u_{i}\right\rangle(\vec{r}) \\
+\frac{1}{2}\left\langle\left[\partial_{\alpha}+\partial_{\alpha}^{+}\right] \cdot\left[u_{\alpha}+u_{\alpha}^{+}\right]\left(\delta u_{i}\right)^{2}\right\rangle \\
+2 / \rho\left(\partial_{i}+\partial_{i}^{+}\right)\left\langle\delta p \cdot \delta u_{i}\right\rangle(\vec{r})+\frac{\partial}{\partial r_{\alpha}}\left\langle\delta u_{\alpha}\left(\delta u_{i}\right)^{2}\right\rangle(\vec{r})= \\
+2 v \frac{\partial^{2}}{\partial r_{\alpha}{ }^{2}}\left\langle\left(\delta u_{i}\right)^{2}\right\rangle(\vec{r})-2\left(\langle\epsilon\rangle+\langle\epsilon\rangle^{+}\right) .
\end{array}
$$

Note that the first four terms correspond to large-scale effects. In (21), each term depends on the spatial vector $\vec{r}$. The equation can be rewritten in a slightly simplified form

$$
\begin{array}{r}
D(\vec{r})+T(\vec{r})+P(\vec{r})+T D(\vec{r})+P D(\vec{r}) \\
+\frac{\partial}{\partial r_{\alpha}}\left\langle\delta u_{\alpha}\left(\delta u_{i}\right)^{2}\right\rangle(\vec{r})=2 v \frac{\partial^{2}}{\partial r_{\alpha}^{2}}\left\langle\left(\delta u_{i}\right)^{2}\right\rangle(\vec{r})-2\left(\langle\epsilon\rangle+\langle\epsilon\rangle^{+}\right),
\end{array}
$$

where terms $D, T, P, T D$, and $P D$ are the decay, transport, production, turbulence diffusion, and pressure diffusion terms, respectively.

An anisotropic (axisymmetric) treatment is to be further applied to Eq. (22), in which each term depends on the spatial vector $\vec{r}$. Note also that this is a scalar equation, representing the scale-by-scale kinetic energy budget. We assume that the flow is axisymmetric with respect to a direction specified by $\vec{n}$. Flow statistics are then invariant to rotations in planes normal to $\vec{n}$ and symmetric with respect to $\vec{n}$. We note

$$
r^{2}=\vec{r} \cdot \vec{r} \quad \text { and } \quad r \mu \equiv \vec{r} \cdot \vec{n}
$$

In this context, each term of the equations depends on two variables, $r$ and $\mu$. Large-scale inhomogeneous variations may be present along the axisymmetry direction $\vec{n}$. We consider that statistics are homogeneous in planes perpendicular to $\vec{n}$.

The advection term $\frac{\partial}{\partial r_{\alpha}}\left\langle\delta u_{\alpha}\left(\delta u_{i}\right)^{2}\right\rangle(\vec{r})$ is written in a manner similar to Refs. 17 and 18. The Laplacian term can be written by using its axisymmetric form (e.g., Ref. 19). After some manipulations, the final axisymmetric form of the scale-by-scale energy budget equation is ${ }^{16}$

$$
\begin{array}{r}
G(r, \mu)=-\frac{2}{3}\left[\langle\epsilon\rangle+\langle\epsilon\rangle^{+}\right]+ \\
2 \nu \frac{1}{r^{3}} \int_{0}^{r} s^{2} \Delta(r, \mu)\left\langle\left(\delta u_{i}\right)^{2}\right\rangle \mathrm{d} s \\
-\frac{1}{r^{3}} \int_{0}^{r} s^{2}(D(r, \mu)+T(r, \mu)+P(r, \mu)+T D(r, \mu)+P D(r, \mu)) \mathrm{d} s,
\end{array}
$$

where $\Delta$ represents the Laplacian. ${ }^{19}$ 
Note the generality of (24) vis à vis the well known, classical "4/3" equation. The main difference is that the axisymmetric equation contains an explicit spatial variation of the mean kinetic energy dissipation rate.

Equation (24) may be expressed in the following, simpler form

$$
\nu F_{1}(r, \mu)+\frac{2}{3}\left(\langle\epsilon\rangle+\langle\epsilon\rangle^{+}\right)+F_{4}(r, \mu)+\operatorname{LST}(r, \mu)=0,
$$

where the function $F_{1}$ involves the Laplacian and $\langle\epsilon\rangle^{+}$indicates the mean energy dissipation rate at the point $\vec{x}+\vec{r}$. For sufficiently small scales, term $F_{4}$ becomes negligible with respect to the first two terms. If LST is negligible, then the only relevant physical parameters are once again $\langle\epsilon\rangle,\langle\epsilon\rangle^{+}$ (the mean energy dissipation rate at the two points) and $v$. Under these conditions, Eq. (25) reduces to

$$
\frac{2}{3}\left[\langle\epsilon\rangle+\langle\epsilon\rangle^{+}\right]=2 \nu \frac{1}{r^{3}} \int_{0}^{r} s^{2} \Delta(r, \mu)\left\langle\left(\delta u_{i}\right)^{2}\right\rangle \mathrm{d} s .
$$

Note again that both members depend on $\vec{r}$, so on $r$ and $\mu$. In this case, it is necessary that the similarity scales $u_{0}$ and $l_{0}$ depend on the angle $\mu$. Rendering dimensionless the two terms with respect to these (as yet unknown) variables leads to

$$
\frac{v}{\langle\epsilon\rangle+\langle\epsilon\rangle^{+}} \cdot \frac{u_{0}^{2}}{l_{0}^{2}}=\operatorname{Const}(\mu)
$$

Now, there are two possibilities:

(i) Along any radial direction (i.e., for any $\mu$ ), the variations of $\langle\epsilon\rangle$ are slow over the dissipative range scales (where the large-scale terms have already been neglected), then along that direction an appropriate set of normalization scales is $u_{K}$ and $\eta$ calculated using $v$ and $\langle\epsilon\rangle$. Note that this requirement, i.e., local homogeneity along any direction defined by $\mu=$ const., is less stringent than that of local isotropy. It is important to stress here that most of the Kolmogorov similarity tests are performed using experimental data obtained with hot/cold wires. Therefore, the spatial scales (or wavenumbers) are almost invariably constructed by assuming the validity of Taylor's hypothesis. The latter, which cannot be circumvented in most cases, has however the disadvantage that homogeneity is artificially introduced along the direction of the mean flow. Under these conditions, the Kolmogorov similarity has the best prospect of being valid in flows where statistics are calculated with Taylor's hypothesis (along the direction of the mean flow), even though those flows are basically anisotropic.

(ii) Another possibility is to calculate statistics from real, spatial information (gathered through either DNS or, e.g., PIV). In these flows, for any value of the angle $\mu$, the variations of $\langle\epsilon\rangle$ are to be taken into account. One solution is to consider the scale $\mathcal{L}$ below which terms LST become negligible. With $\widetilde{\langle\epsilon\rangle}=\left[\langle\epsilon\rangle_{r=0}+\langle\epsilon\rangle_{r=\mathcal{L}}\right] / 2$, the appropriate scales are $\eta=\left(\nu^{3} / \widetilde{\langle\epsilon\rangle}\right)^{1 / 4}$ and $u_{K}=(v \widetilde{\langle\epsilon\rangle})^{1 / 4}$. Again, Kolmogorov scaling is appropriate for any value of the angle $\mu$, and for scales below which the local homogeneity holds.

To conclude this section, we recall that the Kolmogorov similarity does not necessarily require the Reynolds number to be large or local isotropy to be strictly satisfied. A much less restrictive requirement is that the large-scale terms are small compared to the two terms containing $\langle\epsilon\rangle$ and $\nu$ and that local homogeneity along any given $\vec{r}$ direction holds, at least approximately.

\section{RESULTS PERTAINING TO THE COLLAPSE OF KOLMOGOROV-NORMALIZED SPECTRA}

Although the discussion in Secs. II and III has focused on structure functions, we switch our attention here to the spectrum, mainly because of the prevalence of Fig. 1 in the literature; we note however that the collapse of $\left\langle\left(\delta u^{*}\right)^{2}\right\rangle$ in the dissipative range has also been documented, e.g., Fig. 2 of Ref. 20. Figure 1, reproduced from Ref. 21 and based largely on Ref. 22, displays spectra of $u$ (note that $\int_{0}^{\infty} E_{11}\left(k_{1}\right) d k_{1}=\left\langle u^{2}\right\rangle$, where $E_{11}\left(k_{1}\right)$ represents the spectral density of $u$ and $k_{1}$ is the one-dimensional wavenumber along the flow direction) normalized by the Kolmogorov scales $u_{K}$ and $\eta$. An asterisk denotes normalization by $\eta$ and $u_{K}$. The spectra were obtained from a wide variety 


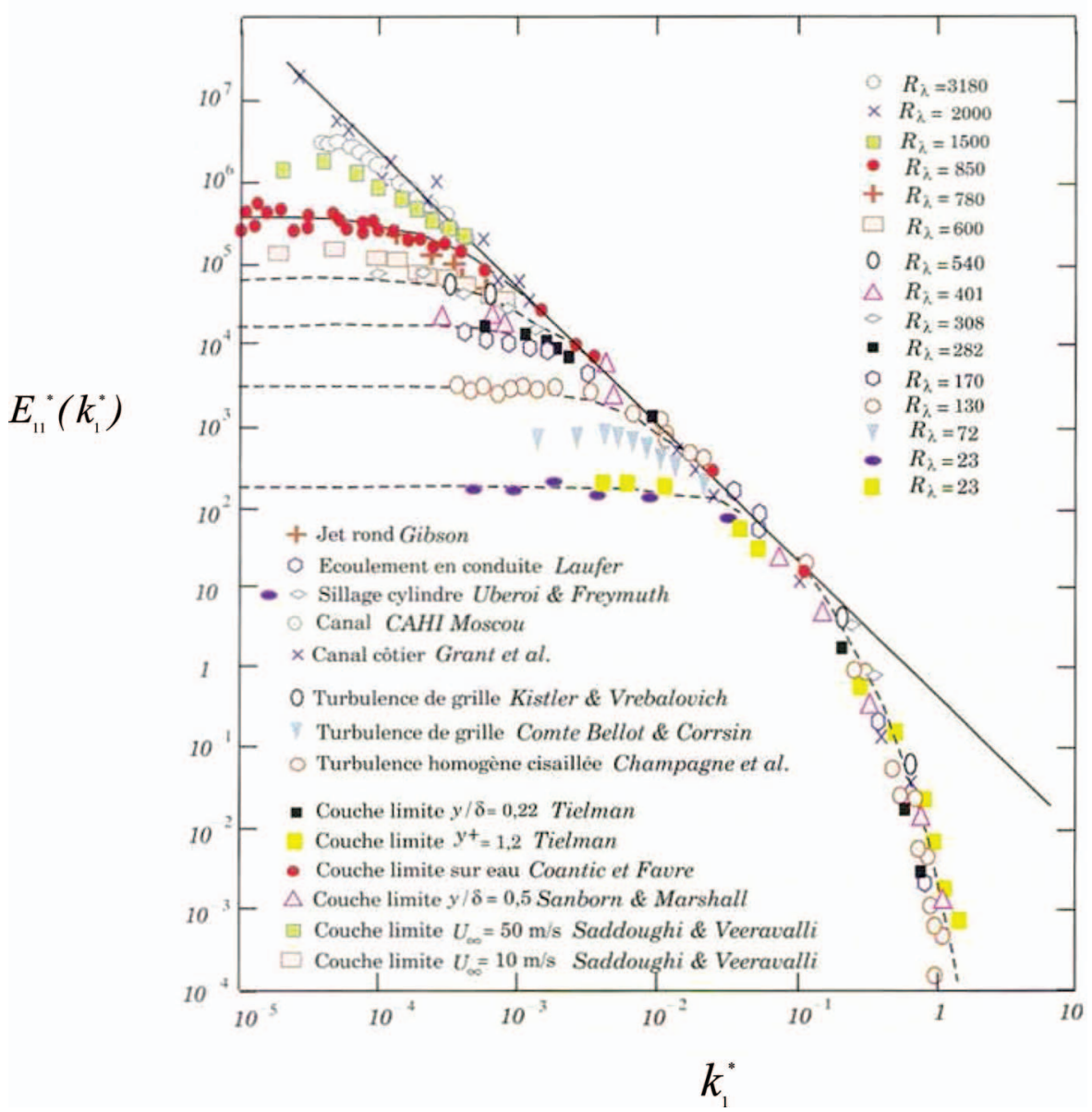

FIG. 1. One-dimensional longitudinal velocity spectra measured in various turbulent flows where $R_{\lambda}$ varies from 23 to 3180 . Adapted and used with the permission of P. Chassaing (Ref. 21, p. 295).

of flows and a wide range of $R_{\lambda}$. The original version of this figure was compiled by Ref. 23 who noted that at small $k_{1}^{*}$, the energy spectrum varies both with $R_{\lambda}$ and type of flow, whereas the small energy dissipating eddies (large $k_{1}^{*}$ ) seem to be universal, i.e., independent of both $R_{\lambda}$ and type of flow. Saddoughi and Veeravalli ${ }^{22}$ remarked that the extent of the IR increases with Reynolds number. While the establishment of a SR with a power-law exponent which tends to $-5 / 3$ as $R_{\lambda}$ increases is unmistakable, the evidence for a $-5 / 3$ range, even for the relatively large value $(\approx 1450)$ of $R_{\lambda}$ in Saddoughi and Veeravalli's boundary layer data, is not entirely convincing. This is reflected in the behaviour of their data for $\left\langle(\delta u)^{3}\right\rangle$ which do not conform rigorously with the "4/5" law. Figure 1 has been used inter-alia in the texts by Refs. 21,24, and 25 mainly in the context of providing support for K41.

It should be noted that Ref. 26 (hereafter GS) also collected measured spectra of $u$ (Fig. 3 of their paper) using the same coordinates as in Fig. 1. Except for the high $R_{\lambda}$ tidal channel data of Ref. 27, all the measurements obtained in grid turbulence with air or water as the fluid were for relatively small values of $R_{\lambda}$. Ref. 26 concluded that the collapse in the DR of all the spectra was quite good, thus providing validation for Kolmogorov's first similarity hypothesis. Figure 3 of Ref. 26 has appeared in the texts of Refs. 28 and 29. Reference 28 noted that it was interesting that the collapse in the DR applied even at values of $R_{\lambda}$ for which there ought to be significant overlap between the energy containing range and the dissipative range. Ref. 26 had previously argued that provided the wavenumber of energy-containing eddies is smaller than $0.2 k_{s}\left(k_{s} \equiv \eta^{-1}=\left(\langle\epsilon\rangle / v^{3}\right)^{1 / 4}\right.$, in the DR, $k_{1}$ is typically greater than $0.1 k_{s}$ ), the DR should in essence be immune from the FRN 


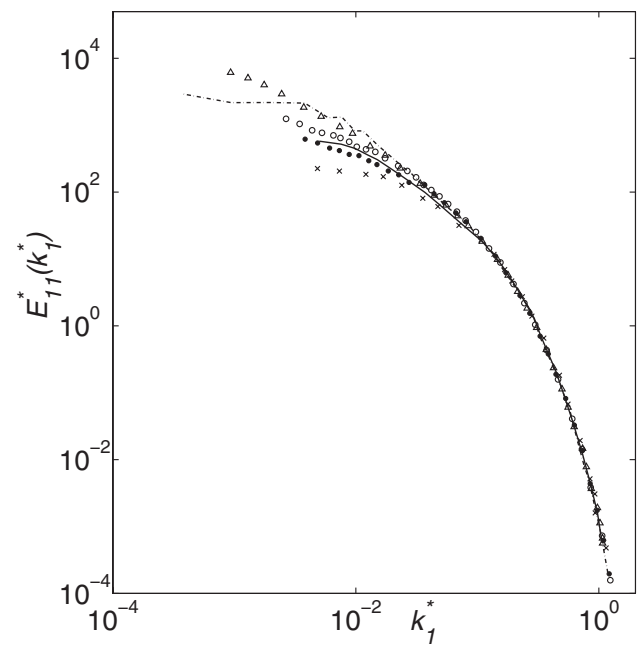

FIG. 2. One-dimensional longitudinal spectra, normalized by Kolmogorov scales, for several turbulent flows and values of $R_{\lambda}$ extending up to about 140. x: Grid turbulence, Ref. 40 (hereafter denoted CBC): $R_{\lambda}=41$; solid line: Grid turbulence, $\mathrm{CBC} R_{\lambda}=65$. Dashed-dotted line: Homogeneous shear flow $\left(R_{\lambda}=130\right)$. Triangles: axis of circular cylinder wake $\left(R_{\lambda}=138\right)$. Both sets of data are adapted from Ref. 41 . Channel flow centreline ${ }^{42}$; open circles: $R_{\lambda} \approx 89$; filled in circles: $R_{\lambda} \approx 67$.

effects. Ref. 29 noted that the collapse of the spectra in the DR, as exhibited, for example, in Fig. 3 of Ref. 26, is not unique as other possibilities, such as the multifractal model and the variational approach of the small scale intermittency (e.g., Refs. 30-32), can result in a collapse of the spectra that is equally as good, if not better than that in Fig. 3 of Ref. 26, or Fig. 1. Ref. 33 also acknowledged that the measured spectra can satisfy other scalings, not consistent with K41, but emphasized the Kolmogorov scaling is the simplest of all available proposals and there are no data which definitely contradict it. We recall here that the analytical considerations of Secs. II and III provide theoretical justification for normalizing the high wavenumber spectrum by Kolmogorov scales. In contrast to $\mathrm{K} 41$, these considerations do not require $R_{\lambda}$ to be large or local isotropy to be satisfied closely.

Leaving aside the important issue of the FRN effect on the SR, the reasonable collapse of the spectra at sufficiently large values of $k_{1}^{*}$, say $k_{1}^{*} \sim 0.1$, strongly implies that K41 should be verified more convincingly by the dissipative scales as $R_{\lambda}$ continues to increase.

It is important to recognise that, as for Fig. 3 of Ref. 26, Fig. 1 contains several spectra for which $R_{\lambda}$ is small; specifically, in seven cases, $R_{\lambda}$ is smaller than 200 . One is therefore led to the

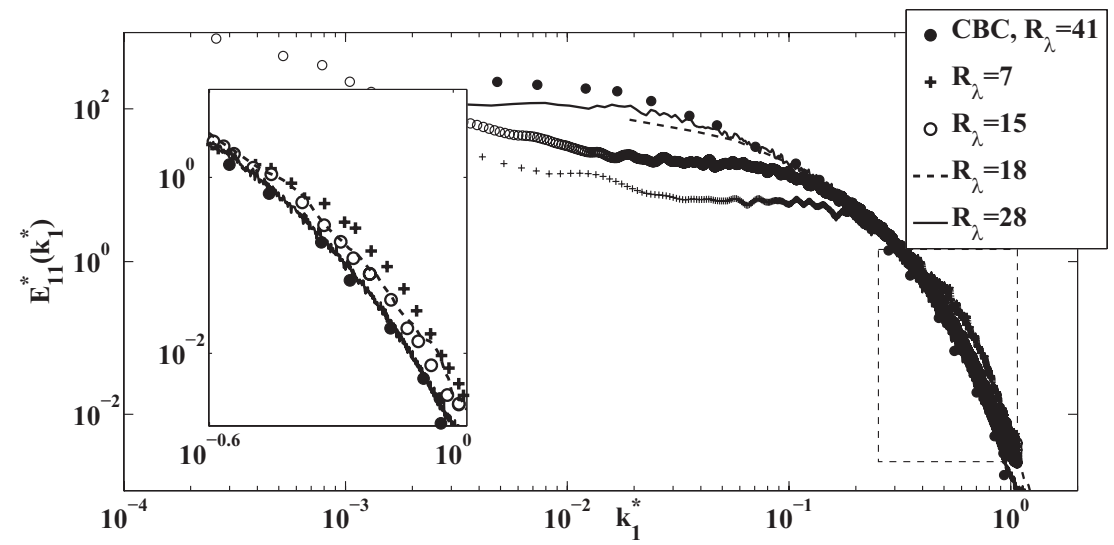

FIG. 3. One-dimensional longitudinal spectra, normalized by Kolmogorov scales, for grid turbulence at low $R_{\lambda}$. CBC: $R_{\lambda}$ $=41$; Present measurements: $R_{\lambda}=28, R_{\lambda}=15, R_{\lambda}=7$. Present DNS: $R_{\lambda}=19$. The inset corresponds to the area enclosed within the dashed rectangle; for clarity, only a few points are shown for the experimental data at $R_{\lambda}=15$ and 7 . 
conclusion that the large $R_{\lambda}$ requirement of K41 is not necessary for the DR to scale in conformity with K41. Two main comments can be made with respect to the collapse in the DR of Fig. 1.

It could be argued that as $k_{1} \eta$ approaches 1 , the collapse is imperfect. The scatter in this range, which is reduced by the use of the logarithmic scales, most likely reflects various measurement difficulties, the most important of these being the onset of electronic noise and the imperfect temporal and spatial resolutions of the probes.

Inevitably, errors have been committed due in part to the difficulties raised under the previous item, but also because of the uncertainty about the actual value of $\langle\epsilon\rangle$ which should be used. For the grid turbulence data examined by Ref. 26, the "true" value of $\langle\epsilon\rangle$ was used for all the spectra obtained in grid turbulence. For this flow, the one-point energy budget is given, to a close approximation, by

$$
\langle\epsilon\rangle=-\frac{U}{2} \frac{d\left\langle q^{2}\right\rangle}{d x}
$$

where $U$ is the mean velocity and $\left\langle q^{2}\right\rangle$ is the mean turbulent energy, equal to $3\left\langle u^{2}\right\rangle$ if isotropy is satisfied by all scales. For grid turbulence, $\langle\epsilon\rangle$ is essentially equal to $\langle\epsilon\rangle_{i s o}$, the isotropic value of $\langle\epsilon\rangle$, viz.,

$$
\langle\epsilon\rangle=\langle\epsilon\rangle_{i s o}=15 v\left\langle\left(\frac{\partial u}{\partial x}\right)^{2}\right\rangle .
$$

Antonia et al. ${ }^{34}$ found that, although the components of the tensor $\left\langle\epsilon_{i j}\right\rangle$ exhibit departures from isotropy, these are generally small, and are of either sign so that Eq. (29) is not invalidated.

Gibson and Schwartz ${ }^{26}$ (also Ref. 28) argued that in fact the true $\langle\epsilon\rangle$ for the grid turbulence data should be replaced by a corrected value, $\left\langle\epsilon_{1}\right\rangle$ say. The latter should correspond to the dissipation rate in flows with very large $R_{\lambda}$ for which the high wavenumber part of the spectrum coincides with that measured in the various grid turbulence flows. Both ${ }^{26,28}$ concluded that the difference between $\langle\epsilon\rangle$ and $\left\langle\epsilon_{1}\right\rangle$ is likely to be small (typically no bigger than $10 \%$ ) and hence indiscernible on a log-log representation, such as that of Fig. 1, since the normalizations of $E_{11}\left(k_{1}\right)$ and $k_{1}$ involve $\langle\epsilon\rangle$ raised to the power of 1/4. In the analysis in Sec. II, which pertains to homogeneous isotropic turbulence, the relevant $\langle\epsilon\rangle$ can only be $\langle\epsilon\rangle_{\text {iso }}$, as given by Eq. (29). In this context, the value of $\langle\epsilon\rangle_{\text {iso }}$ which was used for all the grid turbulence data in Fig. 3 of GS and Fig. 1 seems justifiable.

This raises however the interesting issue of the role the energy cascade, as predicated by $\mathrm{K} 41$, plays in grid turbulence for relatively small $R_{\lambda}$ given that the small scales are approximately isotropic irrespectively of whether a secondary contraction is used downstream of the grid. Antonia et al. ${ }^{35}$ found that isotropy is most closely approximated at all scales when the contraction is used in combination with a grid designed to attenuate vortex shedding from the grid cylinders. For this latter case, the representation illustrated in Fig. 2 is entirely pertinent and $\langle\epsilon\rangle_{\text {iso }}$ is the relevant quantity to be used for normalizing the spectra. Equation (29) can be also written as

$$
\langle\epsilon\rangle=\langle\epsilon\rangle_{\text {iso }}=15 v \int_{0}^{\infty} k_{1}^{2} E_{11}\left(k_{1}\right) d k_{1}
$$

or alternatively

$$
\int_{0}^{\infty} k_{1}^{* 2} E_{11}^{*}\left(k_{1}^{*}\right) d k_{1}^{*}=\frac{1}{15}
$$

Arguably, this latter relation may be thought of as a constraint which partly contributes to the collapse of the spectra in the DR (e.g., Refs. 20 and 36).

To circumvent the criticism raised in the previous paragraph related to the shortcomings of the data as $k_{1} \eta$ approaches 1 , we focus here only on data which are free from the limitations due to the noise and spatial resolution of the measuring sensors. Although corrections for spatial resolution based on Wyngaard' ${ }^{37}$ method can be made, it is difficult to avoid the bias that the key assumptions of this procedure, i.e., the choice of the 3D energy spectrum and the assumption of local isotropy, can introduce in the context of assessing the scaling in the DR. An obvious choice is to use well-resolved DNS data. For suitably chosen values of the grid Reynolds number $U M / v$ ( $M$ is the mesh size of 
the grid) and the distance downstream of the grid, $\eta$ can be close to $1 \mathrm{~mm}$ and hence the length $l_{w}$ of a hot wire, when the latter is used, can be comfortably smaller than $\eta$. For nanoscale thermal anemometry probes or NSTAP (see Ref. 38), $l_{w}$ is currently about $60 \mu \mathrm{m}$ and hence the issue of spatial resolution becomes redundant.

Since two of the data sets in Fig. 1, the DNS data ${ }^{39}$ at the centreline of a channel flow and the grid flow data of Ref. 40 meet the previous requirements, this mitigates to some extent the criticism that the collapse in the DR is contrived.

In his study of small-scale turbulence, Ref. 41 ruled out the use of data obtained with probes of spatial resolution much greater than $\eta$ and for which the low-pass filter cut-off frequency was equal to or smaller than the Kolmogorov frequency. In Fig. 1 of his paper, he plotted spectra of $u$ for 3 different flows, at relatively small values of $R_{\lambda}$ which satisfied these criteria. These corresponding distributions, reproduced in Fig. 2, comply with Kolmogorov scaling for $k_{1}^{*} \geq 0.1$. It is important to underline that no corrections at large $k_{1}^{*}$ were applied to any of the spectra in Fig. 2. It should also be noted that isotropy was used, via Eq. (30), to estimate $\langle\epsilon\rangle$ in all cases. While this assumption is not in doubt for grid turbulence (e.g., CBC), notwithstanding departures from individual components of $\langle\epsilon\rangle$ from isotropy, ${ }^{34}$ it may not be quite as accurate for the quasi-homogeneous shear flow.

We recall that Figs. 1 and 2 may not be sensitive to how accurately $\langle\epsilon\rangle$ is determined since the normalization of $k_{1}$ and $E_{11}\left(k_{1}\right)$ involves $\langle\epsilon\rangle^{1 / 4}$.

Leaving aside the uncertainty surrounding the use of $\langle\epsilon\rangle_{i s o}$ in the wake, Fig. 2 provides convincing support for the Kolmogorov scaling of the DR when $R_{\lambda}$ is small. The DNS spectra included in Fig. 2 were obtained at the centreline of a channel flow at $R_{\lambda}=66$ and $90^{43}$ with resolutions in the $x$ direction of about $0.82 \eta\left(R_{\lambda}=66\right)$ and $1.12 \eta\left(R_{\lambda}=90\right)$ and are in close agreement with the measured spectra from $k_{1}^{*} \approx 0.1$ to slightly beyond $k_{1}^{*} \approx 1$.

However, the DNS spectra of Ref. 44 for decaying isotropic turbulence in a 3D periodic box indicate that the Kolmogorov scaling breaks down when $R_{\lambda}$ is smaller than about 20, suggesting a possible minimum value of $R_{\lambda}$, below which the Kolmogorov normalization ceases to hold.

\section{BREAKDOWN IN THE COLLAPSE OF THE SPECTRA AT SMALL $\boldsymbol{R}_{\lambda}$}

The aim of this section is to indicate the minimum Reynolds number below which Kolmogorov scaling is no longer valid. Because we first ought to provide a quantitative criterion of the statement "the Kolmogorov scaling is no longer valid," it is reasonable to consider that the Kolmogorov scaling holds if it applies over a range of scales wider than a few $\eta$. Therefore, the focus in this section will be on the dissipative range. The starting point is Eq. (11), along with the following considerations. In the dissipative range for $r \rightarrow 0$,

$$
\left\langle(\delta u)^{2}\right\rangle \approx\left\langle\left(\frac{\partial u}{\partial x}\right)^{2}\right\rangle r^{2} .
$$

We denote by $r_{t}=N \eta$ the maximum separation in the dissipation range for which the Kolmogorov similarity holds (the subscript " $t$ " stands for "threshold"). Once again, we focus here on the dissipative range in order to capture the minimum Reynolds number for which the Kolmogorov scaling is no longer tenable. This is not at variance with the well-known fact that, for larger and larger Reynolds numbers, the Kolmogorov scaling may be valid over a range of scales wider than the dissipative range.

Therefore, the magnitude of $\left\langle(\delta u)^{2}\right\rangle$ at $r=r_{t}$ is

$$
\left\langle(\delta u)^{2}\right\rangle_{t} \approx\left\langle\left(\frac{\partial u}{\partial x}\right)^{2}\right\rangle(N \eta)^{2} .
$$

Inserting Eq. (33) into (11) leads to the threshold value $\left(R_{\lambda}\right)_{t}$ for $R_{\lambda}$, viz.,

$$
\frac{\left\langle\left(\frac{\partial u}{\partial x}\right)^{2}\right\rangle(N \eta)^{2}}{u_{K}^{2}} \leq \frac{4}{3} \frac{\left(R_{\lambda}\right)_{t}}{\sqrt{15}},
$$


so that

$$
\left(R_{\lambda}\right)_{t} \geq \frac{3 N^{2}}{4 \sqrt{15}} .
$$

The requirement that Kolmogorov similarity holds for scales smaller than $N \eta$ imposes a minimum Reynolds number provided by Eq. (35). For $N=10$ (i.e., Kolmogorov scaling applies at least for the range of scales $r \leq 10 \eta),\left(R_{\lambda}\right)_{t} \geq 20$, in agreement with Ref. 44 .

$\left(R_{\lambda}\right)_{t}$ can also be estimated using a phenomenological argument, albeit with a slightly different result. As noted in Sec. II, $\left\langle u^{2}\right\rangle /\langle\epsilon\rangle$ may be interpreted as a characteristic time of the large scale motion, viz.,

$$
\tau_{L S} \simeq \frac{\left\langle u^{2}\right\rangle}{\langle\epsilon\rangle},
$$

whereas the dissipative range time scale is given by the Kolmogorov time

$$
\tau_{D} \simeq\left(\frac{v}{\langle\epsilon\rangle}\right)^{1 / 2} .
$$

For Kolmogorov scaling to apply, one expects that

$$
\tau_{D} \ll \tau_{L S}
$$

If we relax this to simply a factor of 10 inequality, viz.,

$$
\tau_{D} \simeq \frac{\tau_{L S}}{10}
$$

then

$$
\left(\frac{v}{\langle\epsilon\rangle}\right)^{1 / 2} \simeq \frac{1}{10} \frac{\left\langle u^{2}\right\rangle}{\langle\epsilon\rangle},
$$

which finally leads to

$$
\left(R_{\lambda}\right)_{t} \simeq 10 \sqrt{15} \simeq 39
$$

i.e., almost twice as large as that given by Eq. (35) with $N=10$.

All the spectra in Fig. 3 correspond to relatively small values of $R_{\lambda}$. The largest $R_{\lambda}$ is 41 , which is for the CBC spectrum; this spectrum can serve as yardstick since it also appears in Fig. 2 and is representative of distributions at higher $R_{\lambda}$. Included in Fig. 3 are the spectra downstream $(x / M=31)$ of a grid consisting of floating flat square elements obtained with a lattice Boltzmann simulation $^{45}$ and spectra measured with a single hot wire downstream of two grids, one consisting of a perforated plate (square holes of size $11 \mathrm{~mm}, M=14 \mathrm{~mm}$ ) and the other a mesh of interwoven wires $(d=1 \mathrm{~mm}, M=5 \mathrm{~mm})$.

Since $U$ is small ( $\leq 5 \mathrm{~m} / \mathrm{s})$, the temporal and spatial resolutions of the hot wire are adequate. Indeed, the length of the wire is $1.27 \eta\left(x / M=35, R e_{M}=U M / v=5140\right)$ for the plate and $0.7 \eta(x / M$ $\left.=470, \operatorname{Re}_{M}=4080\right)$ and $0.26 \eta\left(x / M=470, \operatorname{Re}_{M}=1470\right)$ for the mesh. The spatial resolution for the lattice Boltzmann is about $0.78 \eta$ at $x / M=35$ and $R e_{M}=3200$. The inset in Fig. 3 shows that, relative to the CBC distribution, there is a systematic increase in the magnitude of $E_{11}^{*}\left(k_{1}^{*}\right)$ for $k_{1}^{*} \geq 0.3$ as $R_{\lambda}$ decreases. This behaviour is consistent with the DNS 3D spectra in decaying isotropic turbulence shown in Ref. 44 (their Fig. 2) for $R_{\lambda} \simeq 20$ and 16. These Kolmogorov-normalized spectra were above the distribution of CBC $\left(R_{\lambda}=60.7\right)$. Together with Fig. 2 of Ref. 44, the present Fig. 3 suggests that the breakdown in Kolmogorov scaling is likely to occur when $\left(R_{\lambda}\right)_{t}$ is smaller than 30 , since for $R_{\lambda}=30$ the collapse with CBC is reasonable, whereas at $R_{\lambda}=20$, a clear departure from the universal shape of the spectrum is obvious. The threshold is most likely between 20 and 30 and is reasonably predicted by (35) and (41). This is not an unreasonable outcome, given the approximate natures of (32) and (38), together with using a value of 10 for $N$ and in (39). To detect the exact value of $R_{\lambda}$ at which the Kolmogorov similarity breaks down, further numerical simulations are to be performed. 


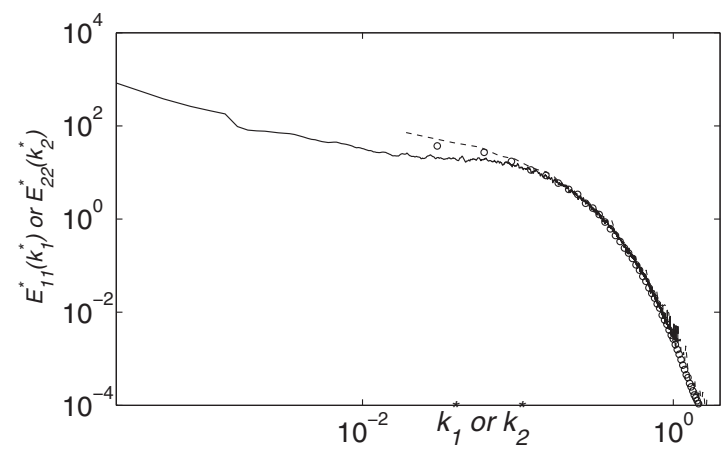

FIG. 4. Kolmogorov-normalized one-dimensional spectra for grid turbulence at $R_{\lambda}=19$ and $x / M=31 .\left(i=1, E_{11}\right.$ : present measurements (solid line), present DNS (dotted line). $i=2, E_{22}$ : present DNS (o).

For decaying isotropic turbulence, it is unlikely that this breakdown is due to a breakdown in local isotropy. Indeed, this is confirmed by Fig. 4 which shows that at $R_{\lambda}=19$, the simulated spectra of $E_{11}^{*}\left(k_{1}^{*}\right)$ and $E_{22}^{*}\left(k_{2}^{*}\right)$ are equal at sufficiently large $k_{1}^{*}$ or $k_{2}^{*}$. This equality is expected if local isotropy applies since $E_{22}\left(k_{2}\right)$ is, like $E_{11}\left(k_{1}\right)$, a "longitudinal" spectra.

\section{CONCLUSIONS}

While Fig. 1 has been used extensively in the literature in support of K41, it more correctly underlines the importance of the FRN effect over the scaling range and a slow approach to an asymptotic state at large values of $R_{\lambda}$. The collapse of the spectra over the dissipative range is in reality convincing only at relatively small values of $R_{\lambda}$, as illustrated in Fig. 2. This collapse supports the premise of Sec. II that a similarity solution for the small scales of motion based on $u_{K}$ and $\eta$ is plausible for decaying-type flows when the effect of the non-stationarity, represented by the $\partial / \partial \mathrm{t}$ term in the KH equation, is negligible. For more complicated flows, such as a quasi-homogeneous shear flow, the Kolmogorov similarity remains plausible provided the inhomogeneity due to the mean shear or mean strain rate does not affect the dissipative scales of motion. In either case, the similarity does not require the restrictions imposed by K41, in particular, the existence of a large separation between the largest scales of the flow and the dissipative scales. Consequently, local isotropy is unlikely to be a stringent requirement, as highlighted in Sec. III.

Kolmogorov similarity does not necessarily require the Reynolds number to be large, or local isotropy to be strictly satisfied. A much less restrictive requirement is that the large-scale terms are small with respect to the two terms containing $\langle\epsilon\rangle$ and $v$, and that local homogeneity along any given direction holds, at least approximately. Kolmogorov similarity has the best prospect of being valid in flows where statistics are calculated with Taylor's hypothesis (along the direction of the mean flow), although these flows are basically anisotropic.

Another conclusion of this paper is that Kolmogorov normalization does break down, even in a shearless flow such as grid turbulence, when $R_{\lambda}$ falls below a certain threshold. Dimensional arguments, supported by numerical and experimental grid turbulence data, suggest that this threshold is between 20 and 30 .

Although the conclusions of the paper may not, at first sight, appear surprising, they do not appear to have yet been enunciated clearly in the literature. It is important that turbulence practitioners are aware of the conditions that lead to a collapse of spectra in the dissipative range, or of those that result in a breakdown of this collapse.

Nonetheless, the robustness of the Kolmogorov normalization at the second-order level is of practical interest. For example, Ref. 46 has put forward the use of a spectral-chart method based on the collapse of the Kolmogorov-normalized spectrum of $u$ in the dissipative range, for estimating $\langle\epsilon\rangle$. 


\section{ACKNOWLEDGMENTS}

We gratefully acknowledge the financial support of the Australian Research Council and Agence Nationale de la Recherche. They also thank Mr. M. D. Kamruzzaman for Figs. 3 and 4.

${ }^{1}$ A. Kolmogorov, "The local structure of turbulence in incompressible viscous fluid for very large Reynolds numbers," Dokl. Akad. Nauk USSR 30, 299 (1941).

${ }^{2}$ J. Qian, "Inertial range and the finite Reynolds number effect of turbulence," Phys. Rev. E 55, 337 (1997).

${ }^{3}$ L. Danaila, F. Anselmet, T. Zhou, and R. A. Antonia, "A generalization of Yaglom's equations which accounts for the large-scale forcing in heated decaying turbulence," J. Fluid Mech. 391, 359 (1999).

${ }^{4}$ E. Lindborg, "Correction to the four-fifths law due to variations of the dissipation," Phys. Fluids 11, 510 (1999).

${ }^{5}$ F. Moisy, P. Tabeling, and H. Willaime, "Kolmogorov equation in a fully developed turbulence experiment," Phys. Rev. Lett. 82, 3994 (1999).

${ }^{6}$ T. Lundgren, "Linearly forced turbulence," CTR Ann. Res. Briefs 461 (2003).

${ }^{7}$ R. A. Antonia and P. Burattini, “Approach to the $4 / 5$ law in homogeneous isotropic turbulence," J. Fluid Mech. 550, 175 (2006).

${ }^{8}$ J. Tchoufag, P. Sagaut, and C. Cambon, "Spectral approach to finite Reynolds number effects on Kolmogorov's $4 / 5$ law in isotropic turbulence," Phys. Fluids 24, 015107 (2012).

${ }^{9}$ A. Kolmogorov, "Dissipation of energy in the locally isotropic turbulence," Dokl. Akad. Nauk. SSSR 32, 19-21 (1941); English translation in Proc. R. Soc. London A 434, 15 (1991).

${ }^{10}$ T. Karman and L. Howarth, "On the statistical theory of isotropic turbulence," Proc. R. Soc. London 164(917), 192 (1938).

${ }^{11}$ G. Batchelor, "Kolmogoroff's theory of locally isotropic turbulence," Proc. Cambridge Philos. Soc. 43, 533 (1947).

${ }^{12}$ L. Landau and E. Lifshitz, Fluid Mechanics, 2nd ed. (Pergamon Press, Oxford, 1987), see also the French translation (Physique Théorique, Tome 6, Mécanique des Fluides).

${ }^{13}$ P. Saffman, "Lectures on homogeneous turbulence," Topics in Non-Linear Physics, edited by N. Zabusky (Springer, 1968), p. 485.

${ }^{14}$ L. Danaila, F. Anselmet, and T. Zhou, "Effect of gradient production on scalar fluctuations in decaying grid turbulence," Flow, Turbul. Combust. 72, 287 (2004).

${ }^{15}$ L. Danaila and L. Mydlarski, "Effect of gradient production on scalar fluctuations in decaying grid turbulence," Phys. Rev. E 64, 016316 (2001).

${ }^{16}$ L. Danaila, J. Krawczynski, F. Thiesset, and B. Renou, "Yaglom-like equation in axisymmetric anisotropic turbulence," Physica D 241, 216- (2012).

${ }^{17}$ G. K. Batchelor, "The theory of axisymmetric turbulence," Proc. R. Soc. London 186, 480 (1946).

${ }^{18}$ B. K. Shivamoggi and R. A. Antonia, "Isotropic and axisymmetric turbulence of passive scalar," Fluid. Dyn. Res. 26, 95 (2000).

${ }^{19}$ S. Chandrasekhar, "The theory of axisymmetric turbulence," Proc. R. Soc. London 242, 557 (1950).

${ }^{20}$ B. Pearson and R. A. Antonia, "Reynolds number dependence of turbulent velocity and pressure increments," J. Fluid Mech. 444, 343 (2001).

${ }^{21}$ P. Chassaing, "Turbulence en mécanique des fluides," Turbulence en Mécanique des Fluides Cépaduès ed. (CEPADUES, 2000).

${ }^{22}$ S. Saddoughi and S. V. Veeravalli, "Local isotropy in turbulent boundary layers at high Reynolds number," J. Fluid Mech. 268, 333 (1994).

${ }^{23}$ D. Chapman, "Computational aerodynamics development and outlook," AIAA Paper No. 17-1293, 1979.

${ }^{24}$ S. Pope, Turbulent Flows (Cambridge University Press, Cambridge, 2000).

${ }^{25}$ P. Davidson, Turbulence (Oxford University Press, 2004).

${ }^{26}$ C. Gibson and W. Schwarz, "The universal equilibrium spectra of turbulent velocity and scalar fields," J. Fluid Mech. 16, 365 (1963).

${ }^{27}$ H. Grant, R. Stewart, and A. Moilliet, “Turbulence spectra from a tidal channel,” J. Fluid Mech. 12, 241 (1962).

${ }^{28}$ A. S. Monin and A. M. Yaglom, Statistical Fluid Dynamics (MIT Press, 2007), Vol. 2.

${ }^{29}$ U. Frisch, Turbulence: The Legacy of A.N. Kolmogorov (Cambridge University Press, 1995).

${ }^{30}$ Y. Gagne and B. Castaing, "A universal representation without global scaling invariance of energy spectra in developed turbulence," C. R. Acad. Sci., Ser. 2 312, 441 (1991).

${ }^{31}$ B. Castaing, Y. Gagne, and M. Marchand, "Log-similarity for turbulent flows?," Physica D 68, 387 (1993).

${ }^{32}$ Z. She and E. Jackson, "On the universal form of energy spectra in fully developed turbulence," Phys. Fluids A 5, 1526 (1993).

${ }^{33}$ M. Nelkin, "Universality and scaling in fully developed turbulence," Adv. Phys. 143-181, 088102 (1994).

${ }^{34}$ R. A. Antonia, T. Zhou, and Y. Zhu, "Three-component vorticity measurements in a turbulent grid flow," J. Fluid Mech. 374, 29 (1998).

${ }^{35}$ R. A. Antonia, P. Lavoie, L. Djenidi, and A. Benaissa, "Effect of small axisymmetric contraction on grid turbulence," Exp. Fluids 49, 3 (2010).

${ }^{36}$ P. Chassaing, R. Antonia, F. Anselmet, L. Joly, and S. Sarkar, Variable Density Fluid Turbulence, Cépadues ed. (Kluwer, 2002).

${ }^{37}$ J. Wyngaard, "Measurements of small-scale turbulence structure with hot wires," J. Phys. E: Sci. Instrum. 1, 1105 (1968).

${ }^{38}$ S. Bailey, G. Kunkd, M. Hultmark, M. Vallikivi, J. Hill, K. Meyer, C. Tsay, G. Arnold, and A. Smits, "Turbulent measurements using a nanoscale thermal anemometry probe," J. Fluid Mech. 663, 160 (2010).

${ }^{39}$ J. Kim and R. A. Antonia, "Isotropy of the small scales of turbulence at low Reynolds number," J. Fluid Mech. 251, 219 (1993). 
${ }^{40}$ G. Comte-Bellot and S. Corrsin, "Simple Eulerian time correlation of full-and narrow-band velocity signals in gridgenerated, isotropic turbulence," J. Fluid Mech. 48, 273 (1971).

${ }^{41}$ F. H. Champagne, "The fine-scale structure of the turbulent velocity field," J. Fluid Mech. 86, 67 (1978).

${ }^{42}$ R. Antonia and H. Abe, "Inertial range similarity for velocity and scalar spectra in a turbulent channel flow," in Proceedings of the Sixth International Symposium on Turbulence, Heat and Mass Transfer, edited by K. Hanjalic, Y. Nagano, and S. Jakirlic (Begell House Inc., 2009), p. 119.

${ }^{43}$ H. Abe, R. A. Antonia, and H. Kawamura, "Correlation between small-scale velocity and scalar fluctuations in a turbulent channel flow," J. Fluid Mech. 627, 1 (2009).

${ }^{44}$ N. Mansour and A. Wray, "Decay of isotropic turbulence at low Reynolds number," Phys. Fluids 6, 808 (1994).

${ }^{45}$ L. Djenidi and S. Tardu, "On the anisotropy of a low-Reynolds number grid turbulence," J. Fluid Mech. 702, 332 (2012).

${ }^{46}$ L. Djenidi and R. A. Antonia, "A spectral chart method for estimating the mean kinetic energy dissipation rate," Exp. Fluids 53, 1005 (2012). 